wave-length and the emitted wave-length, so is the velocity of light to the required velocity, to or from the observer.

A. Cowper Ranyard

\section{PROF. CANNIZZARO'S FARADAY LECTURE} $T$ HIS lecture was delivered on May 30 , by Prof. Chemical Society in honour of the illustrious Faraday, to be held by some eminent foreign savant, who, during the term of his tenure is to deliver a discourse before the Society. Dr. Frankland, in introducing the lecturer, said that in $1869, M$. Dumas had honoured them with his presence there, and on that night they were to listen to Prof. Cannizzaro, of Palermo. After alluding to the numerous investigations which the Professor had made in organic chemistry, and amongst others the discovery of benzylic alcohol, the first normal aromaticalcohol that had ever been prepared, and to the important theoretical views which he had originated, the President, in the name of the Society, presented to him the Faraday Medal, struck in honour of his visit.

Prof. Cannizzaro said that when he received the flattering invitation to deliver the Faraday Lecture, he was placed in very unfavourable circumstances to respond to it, as he had no definite results to lay before the Sosiety, and was, moreover, on the point of suspending his labours and abandoning his old laboratory in order to remove to Rome, and establish a new one there. In this difficulty a subject for a discourse fortunately presented itself, one which the celebrated French chemist, Dumas, had promised to treat of in 1847 , namely, the form which the theory of chemistry should take at the present time. Although this could not be fully discussed in so short a space of time, it would at least have the advantage of directing the attention of chemists to a question of great importance in the transition stage which our science is at present going through.

In recalling the promise which $M$. Dumas had made to the Academy of Sciences of Paris in 1847 , to examine the form which theoretical instruction in chemistry should take in the present state of the science, the lecturer proposed to consider in his discourse the limits within which the exposition of general theories should be included in teaching chemistry, and the form that it was desirable that they should assume. Whilst giving a broad sketch of the progress of modern chemistry, he showed that the atomic theory had become more and more intimately interlaced with the fabric of chemistry, so that it is no longer possible to separate them without rending the tissue, as it were, of the science ; and that up to the present time we have been unable to enunciate even the empirical laws of chemical proportion, independently of that theory; for those who employ the term equivalent in the sense that Wollaston did, commit an anachronism. Consequently, in the exposition of the value and use of symbols, formulæ, and chemical equations, not only are we unable to do without the atomic and molecular theory, but it is inconvenient to follow the long and fatiguing path of induction which leads up to it. By one of those bold flights of the human mind we can at once reach the height whence we discern at a glance the relations between facts.

He then went on to show that the solid basis, the corner-stone of the modern molecular and atomic theory, the crown of the edifice of which Dalton laid the foundation-is the theory of Avogadro and Ampere, Koenig and Clausius, on the constitution of perfect gases, to which chemists, 'Inknown to themselves, have been led in the progress of their science. He thought the time had arrived for reversing the order which had hitherto been followed in teaching chemistry, that instead of setting out from the criteria for determining the weight of mole- cules, and then showing their ratio to the vapour densities, they ought, on the contrary, to commence with the latter, with the theory of Avogadro and Clausius, demonstrating it from physical considerations; to found upon that the proof of the divisibility of simple bodies, that is to say, the existence of atoms; and to show, as occasion presented itself, that the weights of the molecules and the number of the atoms deduced by the application of this theory, are in accordance with those which are deduced from chemical criteria. By this means we can-measure the degree of confidence to be placed in the latter criteria; since so-called compound equivalents do not suffice to determine the weight of molecules, or even to prove their existence, although they may be deduced from a single principle, the theory of the constitution of gases. This is the natural transition from physics to chemistry.

The Professor then stated in detail how he applied the principles he had laid before them. He introduced his pupils to the study of chemistry, by endeavouring to place them on the same leyel as the contemporaries of Lavoisier, and to teach them to appreciate the importance of the principle of the conservation of the weight of matter, showing them that this is quite independent of any idea of its nature or constitution. They are thus led to examine the ponderable composition of substances, so that the student passes rapidly from the epoch of Lavoisier to that of Proust, and then to that of Berzelius at the time when he commenced his researches on proportions. At this stage the same impulse is given to the pupil as Berzelius received on becoming acquainted with the hypothesis of Dalton. The latter is laid before him without any accessory, the use of symbols and formulæ being introduced dogmatically. There will now arise in his mind the same doubts and difficulties that assailed Berthollet, Sir Humphrey Davy, and Wollaston in the application of Dalton's theory, and at the same time a desire for an explanation of the simple relation which exists between the vapour volumes of bodies which react on one another and of the products which are obtained. Now is the moment to state or recall to mind the physical theory of the constitution of the perfect gases. Commencing with a rapid glance at their seneral and specia? chitracters, he insisted, that in this part of the instruction the mind of the student should not be diverted from the numbers expressing their relations, by considerations of the variations caused by changes of temperature and pressure. In applying the theory of the constitution of gases, it will be perceived that the molecules of simple bodies are not always the atoms of Dalton, and a certain confusion will thus be produced in the mind of the beginner in the conception of the ideas of atoms and molecules. The hypothesis of Dalton can now be laid aside, substituting as a starting-point the theory of the relation of molecular weights to the vapour densities. A table must be prepared of the vapour density compared with that of hydrogen as 2, that is to say, the weights of their molecules compared with the weight of the semimolecule of hydrogen taken as unity. We must then compare the composition of the molecules containing the same element-including, or not, the molecule of the element itself-and thence deduce the law of the existence of atoms, that is to say, the amount of each element which always enters, by whole multiples, into the molecules which contain them. We here have the atoms of Dalton which, in the present state of the science, express not only all that Dalton discovered, but also the composition of equal volumes of their vapours, and in the choice of which those doubts can no longer arise which embarrassed Davy and Wollaston. The ideas of molecules and atoms suggested to the student by this law are devoid of all considerations of form, size, continuity, or discontinuity ; the only property indissolubly connected with them is that of ponderability; the very definition of matter. 
Recollecting that no physical theory of the constitution of matter had yet been advanced which thoroughly conformed to chemical ideas, he insisted upon the advisability in teaching the molecular and atomic theory, to keep it free from all that is not absolutely essential, so that it may preserve sufficient plasticity to adapt itself to the progress of our physical and mathematical knowledge. For this purpose he thought it useful to allow the student in the first place to glance at the changes in the hypothesis of the constitution of matter, and then to cause him to estimate the degree of confidence they merit in the actual state of our knowledge. Having thus placed upon a solid basis the fundamental notions of atoms and molecules by the comparison of the composition of equal volumes of the bodies in the gaseous state, it becomes necessary to consider the difficulties which arise in the application of these notions when the vapour densities are wanting; he explained and justified the use of the various auxiliary criteria to which we have resource in these cases, proving them in the first instance by the touchstone of the theory of Avogadro and Clausius, by showing that they gave results in accordance with that theory whenever the two methods can be employed simultaneously.

He believed that we should never lose sight of the starting point, nor give the formulæ of all compounds as of equal probability. "It is not by concealing the obscurity of these questions that we shall enlighten the student ; on the contrary, we should estimate each fact at its true value by showing him that our science does not merit an equal degree of confidence on all points." This forms the introduction, the preparation for the study of the transformations which matter undergoes; the real object and aim of our science.

The comparison of the atomic composition of molecules has led chemists to the law of substitution, to the theory of types of Dumas, then to that of Williamson and Gerhardt, and lastly to the theory of the different valency of atoms and their modes of union, or the socalled theory of atomicity which includes the former. Although at present it is impossible, in teaching chemistry, entirely to eliminate this latter theory, which gives a summary of several laws, and guides us ordinarily in the co-ordination and even prevision of a large number of facts, yet it is difficult to keep it within just bounds so as to avoid infusing into the mind of the beginner illusions which are dangerous for his intellectual education. In order to avoid this, it is advisable to bear in mind the progress of this doctrine and the actual phase of development which it has at present reached. It is still far from being a complete and well-established theory, but is in a state of transition; for although doubtless it embraces a large number of facts, as yet it does not embrace them all. $\mathrm{It}$ is only a partial representation of the reality, and that from a restricted point of view, showing but little relation to our views of the constitution of matter, for it is the result of a comparison of diverse facts expressed by means of the atomic and molecular theory. It is convenient, therefore, to consider each part of this doctrine exclusively in relation to the group of facts which has suggested it.

It is unadvisable to define the valency of atoms as a property inherent in them, and then to deduce as a corollary their different modes of union; on the contrary, it is preferable to regard each portion of this doctrine as a deduction from the observation and comparison of a determinate group of facts, until an opportunity offers to unite these fragments into one whole, not forgetting, however, to notice the gaps which exist, never going beyond what the facts themselves suggest, and never -applying to all bodies indiscriminately, the laws which suit only a single group. For instance, we must not pass over in silence the facts that whilst certain elements are bi tetra- or even hexa-valent, others are tri- and penta-valent; but the pupil should be prevented from acquiring mechanical and geometrical ideas of the cause and effects of the valency of atoms, by frequently reminding him that chemical facts show nothing about the size, form, continuity, or relative position of atoms. If we are sometimes obliged to employ the expression, "relative position of atoms in the molecules," and even to represent them graphically, we must warn the student that these are only artifices to express certain transformations, and that we are really ignorant of the relativei position of the atoms either in space or in the mutual faction of different portions of matter. With these reservations, it is possible, in teaching to derive considerable advantage from/ the theory of atomicity and at the same time to avoid its inconveniences.

In the study of the transformations which matter undergoes, we should direct the pupil's attention, not only to the ponderable changes in the composition of molecules, butalso to the electrical and calorific phenomena which accom pany these transformations. Even from Lavoisier's time it has been recognised that we cannot separate the study of matter from thermic considerations; and every day the connection which exists between chemical and thermic phenomena becomes more apparent.

As in the study of ponderable changes we were guided by the law of the conservation of weight, so in the connection between chemical and dynamical phenomena we are guided by the law of the conservation of force; the two studies mutually supplementing and illustrating one another. Not only will the atomic and molecular theory and that of atomicity help us to compare dynamical phenomena, but the study of dynamical phenomena will show us analogies and differences between chemical actions which would not be observed in the ponderable equations. We should therefore instruct the student in the little definite knowledge which we at present possess concerning thermic and electric phenomena, and especially fix in his mind the fundamental notion of a mechanical equivalent, and the manner of comparing it with chemical action as expressed by the atomic theory. In this we should be aided by the previous or simultaneous instruction of the student in physics under the form and language of the thermodynamic theory.

The lecturer concluded by observing that in the choice of methods and of matter for a course of chemistry, it should always be borne in mind that it was eminently a progressive science, and that even at the time of its most rapid development. The student should start not only with a knowledge of certain definite and fixed principles, but with an aptitude and sufficient preparation to enable him to follow the science in its unceasing transformation and progress, whether he intends expressly to cultivate chemistry, or has only learnt the elements of the science as an auxiliary to other studies or professions. Moueover, the end of chemical instruction for both these classes of students is not only to fix in their memory a certain amount of knowledge, but to assist in their intellectual education. For this, chemistry of all sciences is one of the best, offering both in verbal and practical instructionexcellent occasions for the exercise and harmonious development of all the faculties of the human mind.

He had desired to call attention to what he considered to be the most efficient means of imparting a knowledge of chemistry so that it might serve as an instrument of intellectual education, and that the student, by following it in its ulterior developments, might judiciously apply it to the study of the other branches of natural science. If the attention of the eminent chemists and professors there present were once attracted to this subject, he feit certain that a bright light would be thrown on it, and that our young professors would find numerous suggestions to direct them in teaching chemistry, and that at the very moment when instruction in our science had become so difficult, on account of the rapid transformation which it was undergoing. 
Dr. Williamson said, that those who were there present ought not to separate without some expression of the pleastrre that they had felt on listening to so learned, vast, and eloquent a discourse, treating as it did of a most difficult and important problem. There was scarcely anything of greater moment in the scientific education of youth than the rightly setting before them those wonderful transformations of matter which it is the province of chemistry to explain. These great and growing truths, for, as the lecturer had said, they were growing truths, should be set before youth in such a manner as to form a coherent whole. He hoped to study this masterly discourse with profit and delight, and would now propose a vote of thanks to his illustrious colleague for the honour which he had done them in delivering to them the Faraday lecture.

Prof. Tyndall said he had heard the discourse with deep interest, for it showed that the lecturer knew the importance of a teacher's vocation, and that his province was not merely to communicate knowledge, but to do it in such a manner as to arouse an interest in and love of the subject in the pupil by presenting it in its proper relations. He would have welcomed the lecturer to that Institution, even had he come to tear in pieces the notions which he cherished regarding atoms and molecules; how pleasant it was then to find such a broad agreement between their views. The chemist cannot halt at equivalent proportions - he must ask himself whence they arise, and the inevitable answer is some form of the atomic theory. This theory, however, cannot be confined to chemicalphenomena. The motions of those atoms and molecules underlie all our explanations of the physical cause of light and heat, and it is already taking up the field of magnetism and electricity. Consider, for example, the heat of gases, both as regards the motion of translation of the molecules which produce temperature, and the motions of rotation and vibration of their constituent atoms, which, though they do not express themselves as temperature, constitute a portion of the heat. Clausius has shown that even in the simplest gases nearly two-fifths of the whole heat is due to these internal motions; while in gases of complex molecular constitution which condense on com. bining, the ratio of the total heat to the heat of temperature is still greater. The experiments of Regnauli, which show that the specific heat of a perfect gas at a constant volume is constant, proves, as Clausius has shown, that the one kind of motion is proportional to the others.

The lecturer had also referred to atoms of the same kind combining together, so that, free oxygen and free hydrogen being considered as composed of molecules each containing a pair of atoms, has certainly simplified the results. But it must not be forgotten that this combination of like atoms is widely different from that of unlike atoms. The union of oxygen with oxygen or nitrogen with nitrogen produces no such effects upon the luminiferous ether as the union of oxygen with nitrogen. With the same quantity of matter the amount of vis viva sent forth as radiant heat may be augmented a thousandfold, perhaps a millionfold, by the act of diverse combination. This act seems to carry with it a condensation of the ether to a dense atmosphere around the atoms. Had a cannon the power of gathering round itself a dense atomsphere, it would send forth a greater amount of zis viva as sound. A gun fired at Chamouni may be heard upon Mont Blanc, while the same gun fired on Mont Blanc may not be heard at Chamouni, because the air on which the concussion takes place is denser in the one case than in the other. In the same way the diverse atoms vibrating in the denser atmosphere formed on combination show their vast superiority as radiators over like atoms which, except in such special cases as ozone, \&c., are incompetent to produce a similar condensation. The speaker then asked them to echo the resolution so well put to the meeting by Prof. Williamson.

\section{THE OBSERVATORY ON MOUNT VESUVIUS}

W HILE the scientific world and his own countrymen are rivals in doing honour to Prof. Palmieri for his zeal in remaining at his post in spite of all danger, it may be interesting to examine in some detail the work done at the Observatory of Mount Vesuvius. We know wonderfully little about the origin and mutual dependence of volcanic phenomena. This is due to a want of accurate observations. For the complete investigation we require first to know at what dates earthquakes and eruptions occur at different parts of the earth. Next we must have observations of the direction and exact hour at which a wave of disturbance passes different places whose positions are known. This gives us the velocity of the wave, and helps to determine the position, under the earth's surface, of the centre of disturbance; or, if a wave be propagated over the sea, we obtain a means of estimating the average depth of the intervening ocean ; for the velocity of a wave increases with the depth of the sea. This method gives one of the best determinations we possess of the depth of the Pacific Ocean. But beyond this we must have observations made systematically at some place subject to earthquakes and volcanic eruptions. No place in Europe is more suitable for this than the neighbourhood of Mount Vesuvius ; and it was for such observations that an Observatory was established there.

Everyone knows that Mount Vesuvius consists of a great cone of lava and ashes, at the top of which is the great crater. On the northern side, separated from it by the deep valley called the Atrio del Cavallo, rises the precipitous and semicircular Monte Somma. This once formed the crater of the volcano, and the present cone seems to have been formed inside that great crater at the time when Pompeii was overwhelmed. On a spur of rock, a mile or two in length, running down from the Atrio del Cavallo, the Observatory is placed. It is close to the well-known "Hermitage," or half-way house, in the ascent of the mountain. Being raised on this ridge above the surrounding country, it is comparatively safe from the molten lava that flows at times on either side of it.

The building itself is handsome ; in fact it is to be regretted that so much money should have been devoted to the masonry instead of to additional instruments. On the ground floor are the inhabited rooms, all scantily furnished; but the pursuers of science cannot always expect bodily comfort. On the first floor we find the Museum, with a fire collection of minerals found on the mountain. Perhaps it may be as well here to correct a common mistake as to the nature of the yellow substance found about the craters, whose brilliant colours remind one so much of the Solfatara. This substance is not sulphur, but copper. The most interesting objects in the Museum are the "fumerolles," or smoke-holes. Occasionally at the end of an eruption you may see at the bottom of the crater a small cone of lava, with a hole in its top, through which the steam pours with a hissing noise like a wave breaking on a pebbly beach, or like a blast furnace, or as Pliny has it, like the grinding of a saw; the intensity of the sound varying with your position. These small cones are the fumerolles; they are a foot or two high; and Palmieri has actually had several of these natural chimneys cut off and transported to the Museum.

We now pass on to the Observing Room. There are solid piers carried up from the ground to support the in. struments. First comes the elegant seismograph for the automatic registration of earthquake shocks. The object of the instrument is twofold: first to measure the direction and intensity of a shock; and, second, to write down a history of the earthquake. The shock may be either vertical or horizontal, or partly vertical and partly horizon, tal. For the vertical shocks a fine metallic point is suspended by a coil of wire over a cup of mercury. The coil of wire acts as a spring, and the slightest upward motion of the 